\title{
Celebrating Diversity and Adaptability in Academic Primary Care
}

\section{(Editorial)}

\author{
Lauren Ball $^{1,2}$, Elizabeth Sturgiss ${ }^{3}$
}

${ }^{1}$ School of Allied Health Sciences, Griffith University, Parklands Drive Southport, Gold Coast, QLD 4222, Australia

${ }^{2}$ Menzies Health Institute Queensland, G40 Griffith Health Centre, Level 8.86 Gold Coast campus Griffith University, QLD 4222, Australia

${ }^{3}$ Department of General Practice, School of Primary and Allied Health Care, Monash University, Melbourne, Australia

Corresponding author: Lauren Ball, Mailing Address: Griffith University, N55 2.11, 170 Kessels Rd, Nathan QLD 4111 Australia,Email: 1.ball@griffith.edu.au, Telephone: +61 (0)413 031470 (Mobile), +61 (0)7 37355244 (Work)

Conflict of Interest: LB and ES are Associate Editors for the Australian Journal of Primary Health and are on the Executive Committee of the Australasian Association for Academic Primary Care and Co-Chairs for the 2020 Conference. 
Strong primary care provides healthcare that is efficient, effective and equitable (Starfield et al., 2005). The academic primary care community_including clinician researchers, primary care scientists, professional research staff, and student researchers-are key contributors to developing and disseminating the evidence for primary care. In Australasia, the peak group for the academic primary care community is the Australasian Association for Academic Primary Care (AAAPC).

AAAPC's vision is for a multidisciplinary primary care-oriented health care system founded on patient-centred, primary care principles and developed through evidence and education. The AAAPC will hold its first online Annual Research Conference (ARC 2020) from 14-15 August 2020. It was originally planned to take place on the Gold Coast, hosted by Griffith University, but the global pandemic necessitated a move to a virtual format. The Conference provides an important opportunity to:

- Showcase Australasia's leading primary care research

- Nurture research excellence

- Promote multidisciplinary research networks and collaborations

- Support the translation of evidence into policy and practice

- Strengthen the impact of primary care research.

This issue has the conference abstracts as a supplement (\#reference to supplement). The conference will have over 200 delegates from across Australia, New Zealand and the wider international primary care community. Sessions will include engaging plenary speakers, skills-building workshops and interactive poster sessions. As Conference Chairs, we have 
arranged multiple opportunities for networking in the online space. We are proud of the achievements of the academic primary care community, for the following reasons:

\section{Primary care reimagined}

Patient-centred care is a fundamental component of quality care, but is a relatively modern concept. Conference presentations will advance understanding about how practitioners can listen and act on the needs of patients directly during care as well as how researchers can involve community members in research studies. The ongoing importance of equity and accessibility of services will also be rightly showcased, as well as the values and morals that underpin primary care, including strategies to ameliorate racism and improve equitable access for all people. These presentations will strongly show that as a primary care academic community, we are heading in the right direction by discovering and implementing ways to continuously improve Australia and New Zealand primary care systems and services.

\section{Celebrating diversity}

Primary care embraces the diversity of healthcare needs in our communities and seeks to care for the "whole person". Conference presentations will advance understanding about best practice on a range of clinically relevant topics including contraception care, refugee health, dementia care, diabetes care, smoking cessation, mental health and healthy lifestyle support. This evidence base is important for government policy and a range of organisations working to improve the health of communities, and directly informs the care that clinicians provide for patients through clinical guidelines and practice tips. It is essential that we have evidence that is centred within the context of primary care to shape the future care of patients in the community. 


\section{Learning from each other}

The three plenary presentations will showcase excellence in primary care research, teaching and practice from Australia, New Zealand, UK and North America. We will also hear from research leaders on how the COVID-19 pandemic has influenced primary care, showcasing examples of advocacy, communication and practice that we can all learn from. The social programme will strengthen connections between AAAPC members and show yet again that the primary care academic community is resilient, supportive and genuine in its interest to band together for the purpose of furthering primary care for all.

\section{Strengthening primary care}

The academic primary care community has shown their resolve to continue to connect and share their work despite the limitations brought about by the pandemic. The conference highlights the ways we have re-shaped our research methods to continue building the critical evidence base that will shape the future of healthcare in the community. The AAAPC community welcomes all researchers, educators and clinicians from primary care to join us in advocating for enhanced primary care within Australasia so that health can truly be "for all" (Kluge et al., 2019).

\section{References}

Kluge H, Kelley E, Birtanov Y, et al. Implementing the renewed vision for Primary Health Care in the Declaration of Astana: the time is now. Prim Health Care Res Dev. 2019;20:e137.

Starfield B, Shi L, Macinko J. Contribution of primary care to health systems and health. Milbank Q. 2005;83(3):457-502. 